\title{
Patches of face-selective cortex in the macaque frontal lobe
}

Doris Y. Tsao, Nicole Schweers, Sebastian Moeller, Winrich A. Freiwald

Institute for Brain Research and Center for Advanced Imaging, University of Bremen, P.O.B. 330440, D-28334 Bremen, FR Germany 
In primates, specialized occipital-temporal face areas support the visual analysis of faces, but it is unclear whether similarly specialized areas exist in the frontal lobe. Here, using fMRI in alert macaques, we identified three discrete regions of highly face-selective cortex in ventral prefrontal cortex, one of which was strongly lateralized to the right hemisphere. These prefrontal face patches may constitute dedicated modules for retrieving and responding to facial information. 
Faces are perhaps the most behaviorally significant class of visual forms processed by the primate brain. The perception of a face reveals information about identity, age, gender, mood, gaze direction, and intention, and each of these pieces of information can powerfully influence behavior. Faces activate a wide network of brain areas including the inferior occipital gyrus, fusiform gyrus, superior temporal sulcus, hippocampus, amygdala, inferior frontal gyrus, and orbitofrontal cortex ${ }^{1-5}$. A subset of these areas, all within the ventral pathway dedicated to visual form analysis, has been found to be face selective ${ }^{3,4,6}$ : these areas respond significantly more to faces than to various non-face object categories. In one such face area, the macaque middle face patch, almost all visually responsive cells are face selective ${ }^{7}$.

Since face perception has such a powerful and unique influence on behavior, we wondered whether face-selective modules exist outside the ventral visual pathway for translating face information into behavior. Clusters of face-selective cells have previously been found in prefrontal cortex, in the inferior prefrontal convexity ${ }^{8}$ and in orbitofrontal cortex ${ }^{9}$, but the existence of face areas in prefrontal cortex could not be inferred from these studies due to the low penetration density and the small number of face cells recorded.

We scanned four macaque monkeys using a slice prescription that included all of the frontal lobe. To our knowledge, this is the first attempt to search for face-selective areas in prefrontal cortex of any primate without active task requirements. Previous fMRI studies of face processing either did not systematically cover the frontal lobe ${ }^{4,5,7}$, did not test for face selectivity $1,2,5$, or required performance of cognitive tasks on faces and objects ${ }^{10}$.

Monkeys were scanned while awake and passively fixating. Stimuli consisted of human faces, macaque faces, hands, gadgets, fruits and vegetables, headless bodies, and scrambled patterns, presented in separate blocks. Comparing activation to faces with activation to non-face objects revealed three discrete face-selective patches in prefrontal cortex.

In all four monkeys, we observed a bilateral pair of face patches within the lateral orbital sulcus (Fig. 1a, left column). We term this area PO for "prefrontal orbital". Fig. 1c shows coronal, horizontal, and sagittal slices through the right hemisphere PO of monkey M4. 
We observed a second face-selective patch of cortex on the inferior convexity in all four monkeys (Fig. 1a, middle column). We term this area PL for "prefrontal lateral". A striking feature of PL common to all four monkeys was a strong bias for the right hemisphere. In three animals (M1, M2, M4), PL was found only in the right hemisphere; in the fourth (M3), PL was 2.5 times larger in the right hemisphere than in the left (Supplementary Table 1 gives the size of the face patches in each monkey and hemisphere). PL was located at the base of the inferior convexity in monkeys M1 and M2, and more dorsally, within the fundus of the infraprincipal dimple, in monkeys M3 and M4. This difference in location across animals raises the possibility that PL may actually constitute two functionally distinct regions that are expressed exclusively of each other.

In two animals (M1 and M2), we found a third prefrontal face patch more posteriorly, within the anterior bank of the lower ramus of the arcuate sulcus (Fig. 1a, right column). We term this face-selective region PA for "prefrontal arcuate".

The match between the locations of PL and PO and the previously reported locations of face cell clusters is striking. Scalaidhe et al. ${ }^{8}$ found the largest cluster of face cells in a region within the infraprincipal dimple, precisely where PL was located in monkeys M3 and M4. Rolls et al. ${ }^{9}$ reported a concentration of face cells within the lateral orbitofrontal sulcus, matching the location of PO in all four monkeys. Moreover, the fact that we were able to find these prefrontal face patches in passively fixating animals is consistent with the finding that most prefrontal face cells respond to faces under passive fixation conditions ${ }^{8}$.

In all four monkeys, the existence and location of the face patches was robust across independent scan sessions (as an example, Supplementary Fig. 1a shows the reproducibility of both PO and PL in monkey M4 across two sessions). The overall layout of the three prefrontal face patches was similar across animals (Fig. 1b). Mean time courses (Fig. 1d) and percent signal changes (Fig. 1e) to faces and non-face objects confirm the strong face selectivity of each of the three patches. 
Areas PO, PL, and PA represent a small subset of the larger object-responsive territory in macaque prefrontal cortex. Fig. If shows the activation to faces and non-face objects versus scrambled patterns in two monkeys (M3 and M4). This activation pattern is largely consistent with previous monkey fMRI studies describing activation to objects versus scrambled patterns in a large swath of ventral prefrontal cortex ${ }^{3,11}$. However, activation in orbitofrontal cortex was not reported in a previous monkey fMRI study employing images consisting only of non-face objects ${ }^{11}$.

Orbitofrontal cortex has been implicated in the control of mood, processing of emotions, and social reinforcement ${ }^{12}$, raising the possibility that it may be selective for facial expressions. Thus in a second experiment, we measured responses to neutral and expressive macaque faces (Fig. 2a) and to non-face objects. All three patches responded more strongly to expressive than to neutral faces (Fig. 2b, c). The difference was highly significant in PO $\left(\mathrm{p}=1.9 * 10^{-7}\right)$, but not PL $(p=0.10)$ or PA $(p=0.21)$. In monkey M1, left and right PO were the only two brain regions activated by the contrast (expressive $>$ neutral) at a threshold of $p=0.001$ (Fig. 2d). Temporal lobe face patches, by comparison, were only weakly modulated by facial expression (Supplementary Fig. 2a-c). Thus the increased activity to expressive faces in PO and PA was likely not caused by a general increase in attention or arousal; rather, these areas appear to play a specific role in responding to the emotional content of faces.

Goldman-Rakic and colleagues found face cells with selective delay activity in working memory tasks ${ }^{8}$ near the infraprinciple dimple, where PL was located in two animals. Thus PL may contribute to face-related working memory, attention to faces, face categorization, and perhaps even social reasoning about faces. The most posterior prefrontal face patch, PA, lies at the border between areas 44 and 45B. Area 44 is involved in fine control of facial musculature and may be the macaque homolog of Broca's area ${ }^{13}$. The location of PA near area 44 may facilitate interaction between face perception and control of facial musculature, e.g., in mimicking behavior. PA is also located near area 45B, which is involved in more general cognitive operations including active memory retrieval and set shifting ${ }^{14}$. 
The selective fMRI response to faces in PO, PL, and PA could have been due to specific visual features in faces, to specific internal states elicited by faces (e.g., emotional arousal), or to specific physical behaviors triggered by faces (e.g., lip smacking or eye movements). With the possible exception of PA, it is unlikely that activation of the macaque face patches was due to face-induced physical behaviors, since we did not observe face-selective activations in somatomotor cortex or frontal eye fields. Furthermore, analysis of eye movement traces in the four monkeys did not reveal a significant difference across stimulus conditions (scrambled, faces, objects) for any of five different eye movement parameters analyzed (Supplementary Fig. 3), ruling out differential eye movements as a possible source for the face-selective activation.

Previous work in humans on the material specificity of prefrontal cortex during memory tasks suggests a broad specialization for verbalizable content (e.g., words) in the left and for nonverbalizable content (e.g., faces and abstract patterns) in the right posterior inferior frontal cortex 10. The strong right lateralization of PL raises the possibility that it may constitute the macaque homolog of the region in human right prefrontal cortex activated during remembrance of faces. It is then tempting to ask what is being coded by the corresponding left hemisphere region in the macaque -- the answer may reveal a precursor of language.

The variety of cognitive tasks that involve faces is endless. However, the visual component of each of these tasks may only require communication between prefrontal cortex and face-selective areas of inferotemporal cortex. Therefore, PO, PL, and PA could receive visual inputs primarily from face modules in IT cortex, and at the same time receive diverse inputs from other sensory sources, and send outputs to a widespread region of cortex, as necessary for effecting a rich repertoire of face-related behavioral responses. Our finding of a regional specialization for faces supports the notion put forward by Goldman-Rakic that information domain is a powerful organizing principle in prefrontal cortex ${ }^{8}$.

Acknowledgements: We are grateful to Katrin Thoss and Ramazani Hakizimana for technical support, to Michael Borisov for help with data analysis, to Hidehiko Komatsu for providing 
pictures of macaque faces, and to Guerbet for providing Sinerem. This work was supported by a Sofia Kovalevskaya Award from the Alexander von Humboldt Foundation, by the German Science Foundation (DFG FR1437/3-1), and by the German Ministry of Science (Grant 01G00506, Bremen Center for Advanced Imaging).

\section{Figure legends}

Figure 1. Face-selective patches in prefrontal cortex of four macaques.

a) Face-selective patches from four macaques superimposed on coronal anatomical slices. Activated regions showed a significantly greater response to faces than to four categories of nonface objects. The first column shows the orbital face patch (PO), the second column shows the ventrolateral face patch (PL), and the third column shows the face patch at the ventral tip of the anterior bank of the inferior arcuate sulcus (PA); patches are indicated by white arrows. The AP position of each slice is indicated at the top left corner of each slice (in mm relative to the interaural canal). Sulcal labels for (a) and (b): ps: principle sulcus, al: lower ramus of arcuate sulcus, $s f$ : Sylvan fissure, los: lateral orbital sulcus, ipd: infraprincipal dimple.

b) Prefrontal face patches overlaid on reconstructed inflated left and right frontal lobes of the four monkeys. Order of monkeys from top to bottom same as in (a).

c) Coronal, horizontal, and sagittal slices through the right hemisphere PO of monkey M4.

d) Mean time courses extracted from the face patches shown in (a), averaged across monkeys and across hemispheres for patches that were bilateral. To generate the data shown here and in (e) below, even runs were used to define ROI's for the face patches in each monkey, and odd runs were used to extract the time courses and activation values. Three different visual stimulation conditions were presented: faces (light grey epochs; F: human faces, M: monkey faces), non-face objects (dark grey epochs; H: hands, G: gadgets, V: vegetables and fruits, B: headless bodies), and scrambled versions of the same images (white epochs).

e) Bar graph showing \% fMRI signal change to faces (light grey bars) and to non-face objects 
(dark grey bars) in the three prefrontal face patches. Error bars indicate $95 \%$ confidence intervals.

f) Top: atlas of macaque prefrontal cortex, adapted from Petrides and Pandya ${ }^{15}$. Lateral view on the left, ventral view on the right. The junction between areas 9/46v, 45A, and 47/12 coincides with the anterior end of the infraprincipal dimple (ipd). ala: anterior bank of lower ramus of arcuate sulcus. Bottom: Activation to faces and non-face objects (hands, gadgets, vegetables and fruits, and bodies) versus grid-scrambled patterns. The prefrontal face patches are indicated by green outlines. In both of these monkeys (M3 and M4), the infraprinciple dimple could be seen in the anatomical MR images, and PL was located partially within this dimple. See Supplementary Text 1 for discussion of area assignments for the three prefrontal face patches.

Figure 2. Sensitivity of prefrontal and temporal face patches to facial expression.

a) Example images of neutral and expressive macaque faces used to test selectivity of prefrontal face patches for expressive versus neutral faces.

b) Average time course from PO, PL, and PA to neutral faces (medium grey), expressive faces (light grey), and non-face objects (dark grey). Data averaged across three monkeys (M1, M3, M4).

c) Bar graph showing $\%$ fMRI signal change to neutral faces (medium grey), expressive faces (light grey), and non-face objects (dark grey). Data averaged across three monkeys (M1, M3, M4). Error bars indicate 95\% confidence intervals.

d) Coronal slice showing activation to expressive versus neutral faces in monkey M1. PO was the only region activated by this contrast at $\mathrm{p}=0.001$.

\section{References}


1. Haxby, J.V., Hoffman, E.A. \& Gobbini, M.I. The distributed human neural system for face perception. Trends Cogn Sci 4, 223-233 (2000).

2. Ishai, A., Schmidt, C.F. \& Boesiger, P. Face perception is mediated by a distributed cortical network. Brain Res Bull 67, 87-93 (2005).

3. Tsao, D.Y., Freiwald, W.A., Knutsen, T.A., Mandeville, J.B. \& Tootell, R.B. Faces and objects in macaque cerebral cortex. Nat Neurosci 6, 989-95 (2003).

4. Pinsk, M.A., DeSimone, K., Moore, T., Gross, C.G. \& Kastner, S. Representations of faces and body parts in macaque temporal cortex: a functional MRI study. Proc Natl Acad Sci U S A 102, 6996-7001 (2005).

5. Hoffman, K.L., Gothard, K.M., Schmid, M.C. \& Logothetis, N.K. Facial-expression and gaze-selective responses in the monkey amygdala. Curr Biol 17, 766-72 (2007).

6. Kanwisher, N., McDermott, J. \& Chun, M. The fusiform face area: A module in human extrastriate cortex specialized for face perception. Journal of Neuroscience 17, 43024311 (1997).

7. Tsao, D.Y., Freiwald, W.A., Tootell, R.B.H. \& Livingstone, M.S. A cortical region consisting entirely of face-selective cells. Science 311, 670-674 (2006).

8. Scalaidhe, S.P., Wilson, F.A. \& Goldman-Rakic, P.S. Face-selective neurons during passive viewing and working memory performance of rhesus monkeys: evidence for intrinsic specialization of neuronal coding. Cereb Cortex 9, 459-75 (1999).

9. Rolls, E.T., Critchley, H.D., Browning, A.S. \& Inoue, K. Face-selective and auditory neurons in the primate orbitofrontal cortex. Exp Brain Res 170, 74-87 (2006).

10. Kelley, W.M. et al. Hemispheric specialization in human dorsal frontal cortex and medial temporal lobe for verbal and nonverbal memory encoding. Neuron 20, 927-36 (1998).

11. Denys, K. et al. Visual activation in prefrontal cortex is stronger in monkeys than in humans. J Cogn Neurosci 16, 1505-16 (2004).

12. Rolls, E.T. The brain and emotion, (Oxford University Press, Oxford, 1999).

13. Petrides, M., Cadoret, G. \& Mackey, S. Orofacial somatomotor responses in the macaque monkey homologue of Broca's area. Nature 435, 1235-8 (2005).

14. Nakahara, K., Hayashi, T., Konishi, S. \& Miyashita, Y. Functional MRI of macaque monkeys performing a cognitive set-shifting task. Science 295, 1532-6 (2002).

15. Petrides, M. \& Pandya, D.N. Comparative cytoarchitectonic analysis of the human and the macaque ventrolateral prefrontal cortex and corticocortical connection patterns in the monkey. Eur J Neurosci 16, 291-310 (2001). 
a
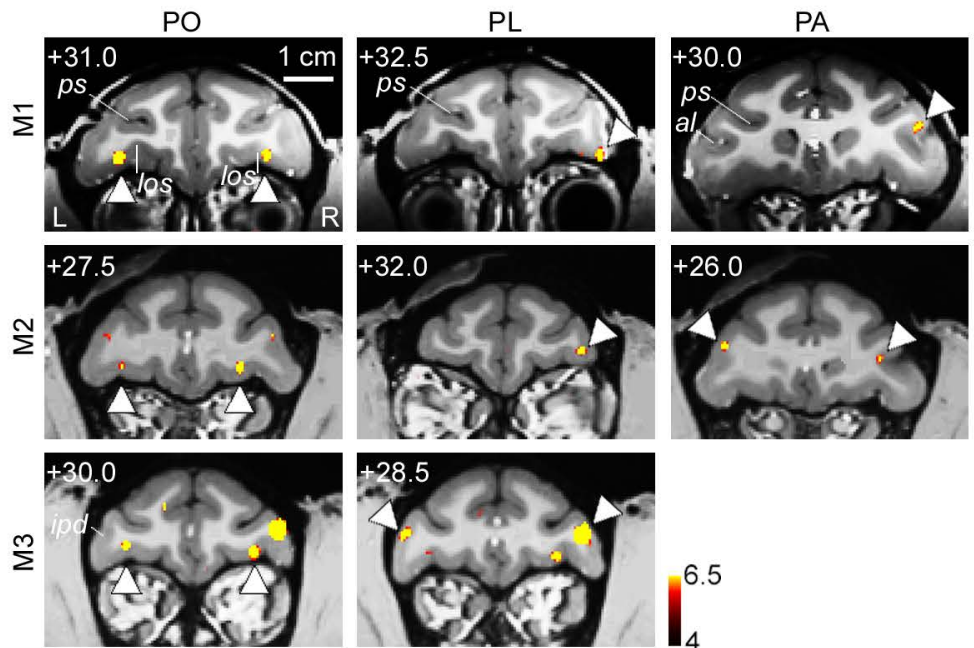

$+28.5$
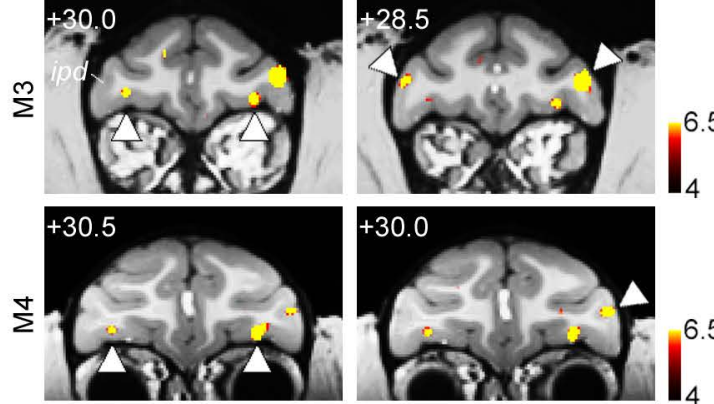

\section{$+30.0$}

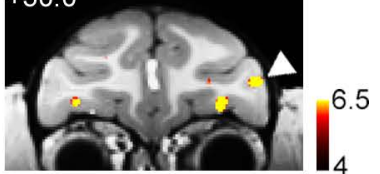

6.5 b
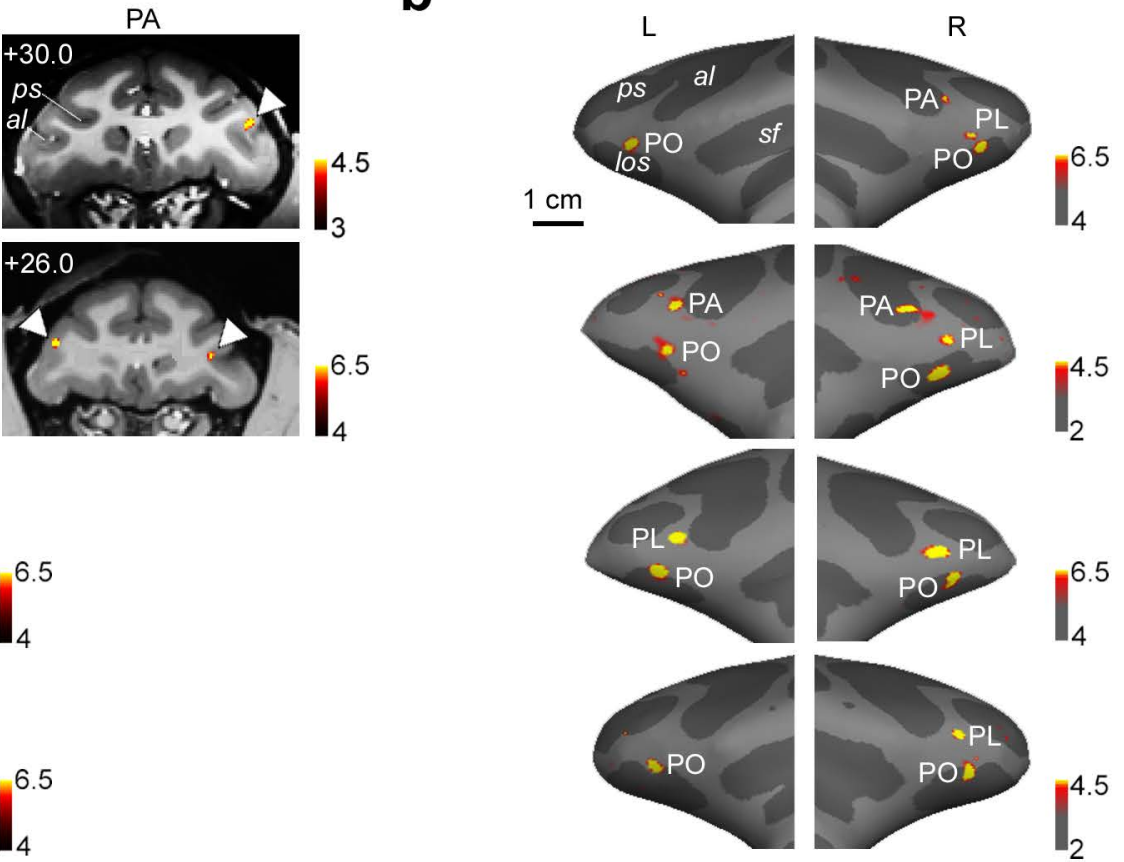

f
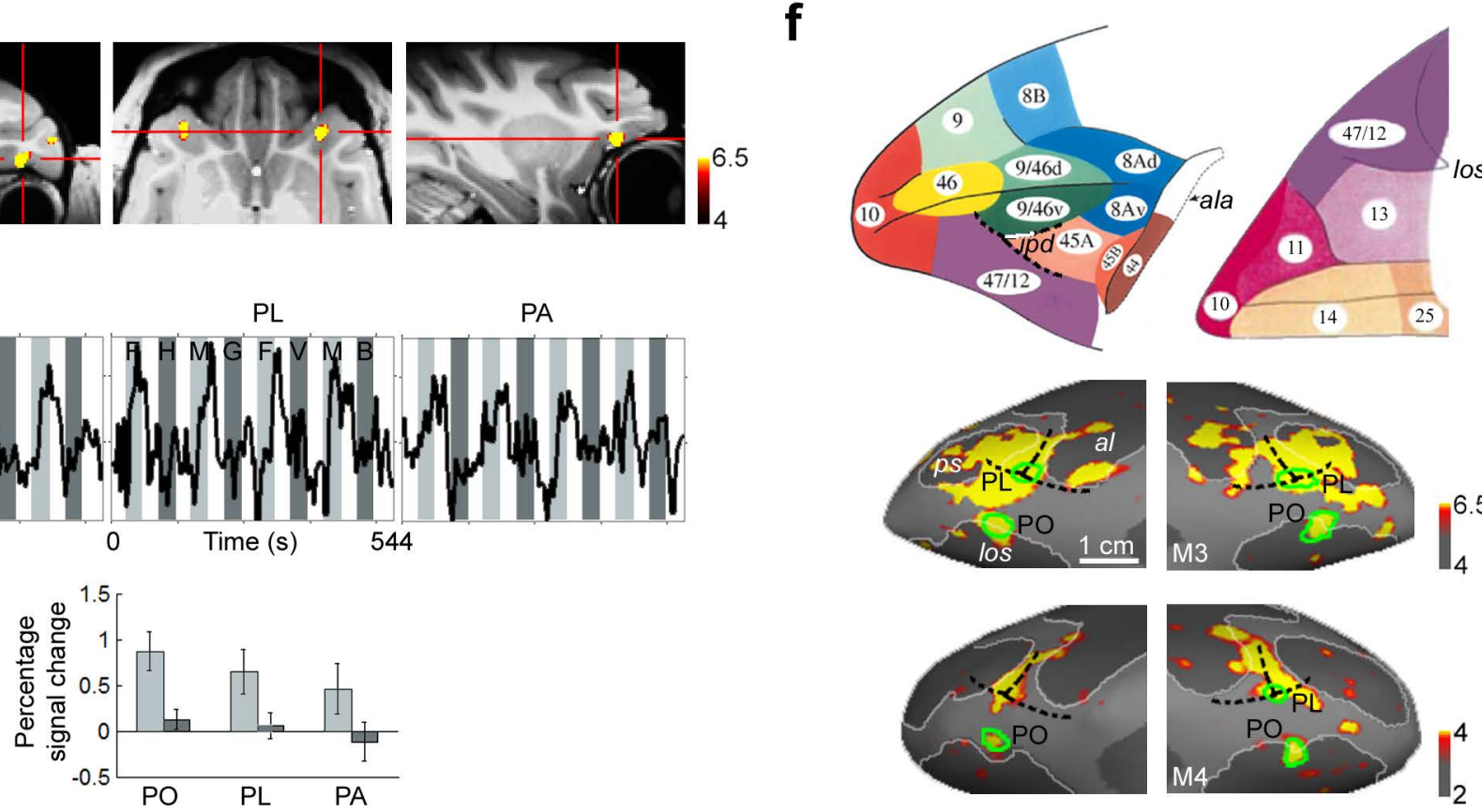

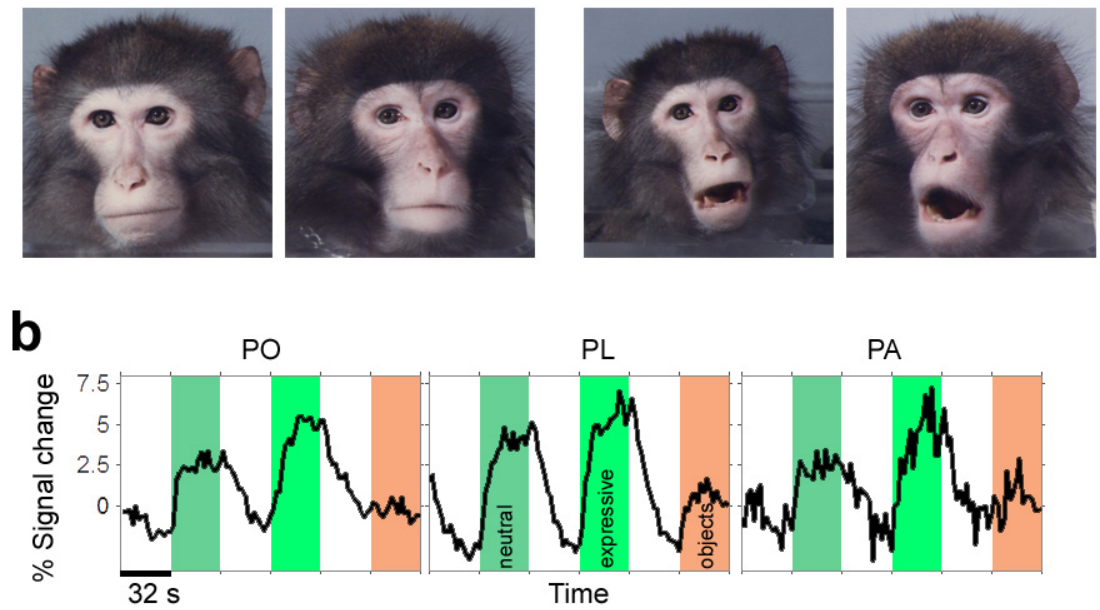

C
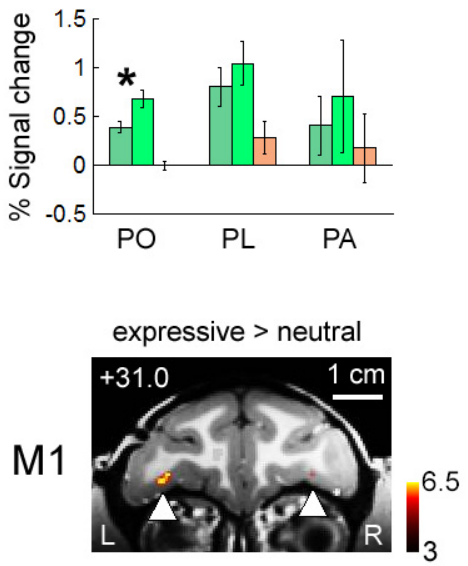